\title{
How can we improve stroke thrombolysis rates? A review of health system factors and approaches associated with thrombolysis administration rates in acute stroke care
}

Christine L. Paul ${ }^{1,3^{*}}$, Annika Ryan ${ }^{1,3}$, Shiho Rose ${ }^{1,3}$, John R. Attia ${ }^{1,3}$, Erin Kerr², Claudia Koller ${ }^{1,3}$ and Christopher R. Levi ${ }^{1,2,3}$

\begin{abstract}
Background: Thrombolysis using intravenous (IV) tissue plasminogen activator (tPA) is one of few evidence-based acute stroke treatments, yet achieving high rates of IV tPA delivery has been problematic. The 4.5 -h treatment window, the complexity of determining eligibility criteria and the availability of expertise and required resources may impact on treatment rates, with barriers encountered at the levels of the individual clinician, the social context and the health system itself. The review aimed to describe health system factors associated with higher rates of IV tPA administration for ischemic stroke and to identify whether system-focussed interventions increased tPA rates for ischemic stroke.

Methods: Published original English-language research from four electronic databases spanning 1997-2014 was examined. Observational studies of the association between health system factors and tPA rates were described separately from studies of system-focussed intervention strategies aiming to increase tPA rates. Where study outcomes were sufficiently similar, a pooled meta-analysis of outcomes was conducted.

Results: Forty-one articles met the inclusion criteria: 7 were methodologically rigorous interventions that met the Cochrane Collaboration Evidence for Practice and Organization of Care (EPOC) study design guidelines and 34 described observed associations between health system factors and rates of IV tPA. System-related factors generally associated with higher IV tPA rates were as follows: urban location, centralised or hub and spoke models, treatment by a neurologist/stroke nurse, in a neurology department/stroke unit or teaching hospital, being admitted by ambulance or mobile team and stroke-specific protocols. Results of the intervention studies suggest that telemedicine approaches did not consistently increase IV tPA rates. Quality improvement strategies appear able to provide modest increases in stroke thrombolysis (pooled odds ratio $=2.1, p=0.05$ ).

Conclusions: In order to improve IV tPA rates in acute stroke care, specific health system factors need to be targeted. Multi-component quality improvement approaches can improve IV tPA rates for stroke, although more thoughtfully designed and well-reported trials are required to safely increase rates of IV tPA to eligible stroke patients.
\end{abstract}

Keywords: Ischemic stroke, Thrombolysis, Implementation, Quality improvement, Health system change, Tissue plasminogen activator

\footnotetext{
* Correspondence: Chris.Paul@newcastle.edu.au

${ }^{1}$ The University of Newcastle, University Drive, Callaghan, NSW 2308, Australia

${ }^{3}$ Hunter Medical Research Institute, 1/Kookaburra Circuit, New Lambton Heights,

NSW 2305, Australia

Full list of author information is available at the end of the article
}

\section{Biomed Central}

C 2016 Paul et al. Open Access This article is distributed under the terms of the Creative Commons Attribution 4.0 International License (http://creativecommons.org/licenses/by/4.0/), which permits unrestricted use, distribution, and reproduction in any medium, provided you give appropriate credit to the original author(s) and the source, provide a link to the Creative Commons license, and indicate if changes were made. The Creative Commons Public Domain Dedication waiver (http://creativecommons.org/publicdomain/zero/1.0/) applies to the data made available in this article, unless otherwise stated. 


\section{Background}

Stroke causes five million deaths worldwide [1, 2] with escalating costs to the health system [3-6]. Most stroke cases (89 \%) are admitted to hospital [7], with approximately $50 \%$ of sufferers left deceased or dependent [8]. Thrombolysis using intravenous (IV) tissue plasminogen activator (tPA) is one of the few evidence-based acute stroke treatments $[9,10]$.

Despite the potential benefit offered by routine delivery of thrombolysis to eligible stroke patients, achieving and sustaining high rates of IV tPA delivery has been problematic.

While seeking treatment late is a major limiting factor on tPA delivery $[11,12]$, health system factors (i.e. circumstances that are determined by the health organisation or the health care provider rather than the individual) are important in improving access to thrombolysis for stroke patients. While there is no agreed benchmark for rates or levels of thrombolysis in practice, substantial change has been shown to be achievable such as an increase in tPA administration rate from 4.7 to $21.4 \%$ of all stroke patients [13].

The narrow treatment window of $4.5 \mathrm{~h}$ from stroke onset, negative impacts of inappropriate treatment, along with the multi-step, multi-disciplinary testing, and decision-making process needed to determine thrombolysis eligibility would indicate that complex interventions are required to change thrombolysis rates [14]. Complex interventions are generally defined as those which involve a number of interacting components, require a number of behaviours or difficult behaviours, involve a number of groups or organisational levels and have a number of outcomes [14], each of which is directly relevant to thrombolysis for acute stroke. Barriers to treatment include delays in stroke recognition by staff [15], delays in obtaining and interpreting radiology imaging [16], inefficiencies in emergency stroke care and delays in obtaining treatment consent [17].

Study of the diffusion of new technologies indicates that while some innovations are largely adopted in less than 5 years [18], others may fail to become commonplace due to barriers or failures at a higher level [19]. In these contexts, the use of theoretical frameworks such as the Behaviour Change Wheel (BCW) [20] can be helpful to clarify the range of factors which may need to be addressed in order to effect change. The BCW describes the three essential conditions for behaviour change to occur: capability, opportunity and motivation; nine intervention functions and seven policy categories are required for whole system change [20]. Models and frameworks such as the BCW emphasise the importance of intervening not only at the level of the individual but also at an organisational or system level and at the broader policy level. While policy-level factors such as financial incentives may impact on thrombolysis over the long term [21], in the short to medium term, health service providers may have the greatest potential impact by acting at a health system or organisational level.

A number of cross-sectional studies have described associations between higher stroke tPA rates and systemlevel factors such as hospital size and hospital type $[22,23]$ or characteristics such as staffing [24] or stroke certification [25-28]. System-level approaches have been recommended to improve access to IV tPA and increase the proportion of patients receiving the treatment, including telemedicine and centralised hub and spoke models [29-31]. Some studies have described successful attempts to apply hospital pre-notification systems [13] or quality-improvement approaches (e.g. analysing performance, with systematic efforts to improve it, ultimately resulting in better health outcomes) [32, 33], to increase tPA implementation for stroke.

However, system changes require substantial resources and engagement with quality improvement programmes. To our knowledge, there are no published reviews of a broad range of evidence-based health system factors associated with increased IV tPA administration rates for stroke.

\section{Aims}

The aim of this study is to identify the following:

- Health system factors associated with higher rates of IV tPA administration for ischemic stroke

- The effectiveness of system-focussed intervention strategies, which meet Cochrane Collaboration Evidence for Practice and Organization of Care (EPOC) study design guidelines, in improving IV tPA rates for treatment of ischemic stroke

\section{Methods}

\section{Search strategy}

The literature review in MEDLINE, CINAHL, EMBASE and PsycINFO spanned from January 1997 to May 2014 and was performed as title, abstract and full-text review by three independent reviewers, with ambiguous articles discussed as a group to reach agreement. The search period was selected to align with the 1996 approval of the "clot-buster" drug [34] and the release of the first tPA stroke guidelines [35]. Search terms were confirmed in consultation with clinical stakeholders and a medical librarian. Available MeSH headings were used; otherwise, a "title" field search was conducted.

Limitations included published original research, English language, humans, adults, and used a combination of keyword searches of "tpa.m_titl" OR "rtpa.m_titl" OR "Tissue Plasminogen Activator OR Tissue Plasminogen Activator.m_titl" OR "Fibrinolytic Agents OR Fibrinolytic 
Agents.m_titl" OR "Recombinant Proteins OR Recombinant Proteins.m_titl" OR "Thrombolytic Therapy OR Thrombolytic Therapy.m_titl" AND "Stroke OR Stroke.m_ title" OR "Brain Ischemia OR Brain Ischemia.m_titl" OR "Cerebral Hemorrhage or Cerebral Hemorrhage.m_titl".

\section{Inclusion criteria}

The inclusion criteria are as follows:

- Studies that quantitatively assessed modifiable health system factors influencing rates of IV tPA for stroke; or

- Intervention studies aiming to improve rates of IV tPA administration for stroke

\section{Exclusion criteria}

The exclusion criteria are as follows:

- Solely addressing patient characteristics such as age, race, education, income or clinical eligibility for thrombolysis

- No denominator for calculating tPA rates or not reporting a tPA rate

- Solely assessing intra-arterial tPA

- Addressing only community-directed or patientdirected activities or changes

- Hypothetical studies

\section{Data extraction}

\section{Health system factors}

Using an extraction template, the following health system factors were extracted: sample characteristics, sample size, response rate, descriptors of setting, data collection method, rate and proportion of IV TPA administration, system factors addressed in relation to tPA delivery, factors affecting IV tPA rates, tPA criteria/ guidelines and TPA time window. Using existing frameworks such as the BCW to categorise the identified health system factors was not successful as a number of the strategies could be categorised as having multiple intervention functions. Only three of the nine intervention functions and two of the seven policy functions described in the $\mathrm{BCW}$ were identified in the review. Therefore, a consensus process was used among the authors to identify practice-relevant categories under which to present the observational studies.

\section{Interventions}

Intervention studies were reviewed and categorised according to whether or not they met criteria for any of the four experimental designs defined and recommended by the EPOC design criteria. Data extracted were as follows: study design; setting; target group; study duration; intervention allocation; unit of analysis; allocation concealment; blinding; eligibility criteria; sample size; representativeness of sample; intervention conditions; outcome measures; statistical analysis; and findings.

\section{Quality control}

Quality control involved second coding of a random sample of articles (10\%) at each review stage, i.e. initial extraction of studies and exclusion of ineligible studies (AR, SR, CP). Extracted data from all included studies were double-coded in full (AR, SR, CP, CK, JA, EK) and checked for agreement (AR). Agreement rates exceeded $90 \%$ at all stages. All remaining differences in inclusions, exclusions and extracted data were discussed according to documented principles until consensus was reached, with subsequent re-coding completed wherever necessary.

\section{Analysis}

For the experimental studies, synthesis of the data involved meta-analysis where possible. Only studies which had pre- and post-test data specifying the rate of thrombolysis for intervention versus control groups were included in the meta-analysis. For the four study outcomes that were sufficiently similar, a pooled meta-analysis of outcomes was conducted using StatsDirect (version 2.7.9., Cheshire, UK). Heterogeneity was checked using $I^{2}$ and if high, random effects (DerSimonian-Laird method) pooling was used. Narrative synthesis was used to describe outcomes for the remainder of the experimental studies which could not be included in the meta-analysis due to heterogeneity of outcomes. Narrative synthesis [36] involved verbal descriptions of the extracted data. For the observational studies, data synthesis involved tabulation of whether the study found a significant association with thrombolysis rate for any review-relevant factor followed by comparative narrative synthesis.

\section{Results}

The search resulted in 4323 citations (MEDLINE $n=1947$, EMBASE $n=1760$, PsycINFO $n=46$, CINAHL $n=570$ ). As indicated in Fig. 1, 34 studies reported associations between health system factors and IV tPA rates for ischemic stroke. Seven intervention studies that reported an improvement in IV tPA rates met the EPOC design criteria. Forty-seven intervention studies were excluded as being either pre-test-post-test designs with no control group or pilot tests with post-test only data. The types of intervention strategies studied in the 47 excluded publications included the following: the introduction of stroke units or "code-stroke" protocols; support for regional sites (e.g. hub and spoke models, telemedicine); changes in hospital protocols, staffing or rostering; and the "Get With The Guidelines" programme [37]. 

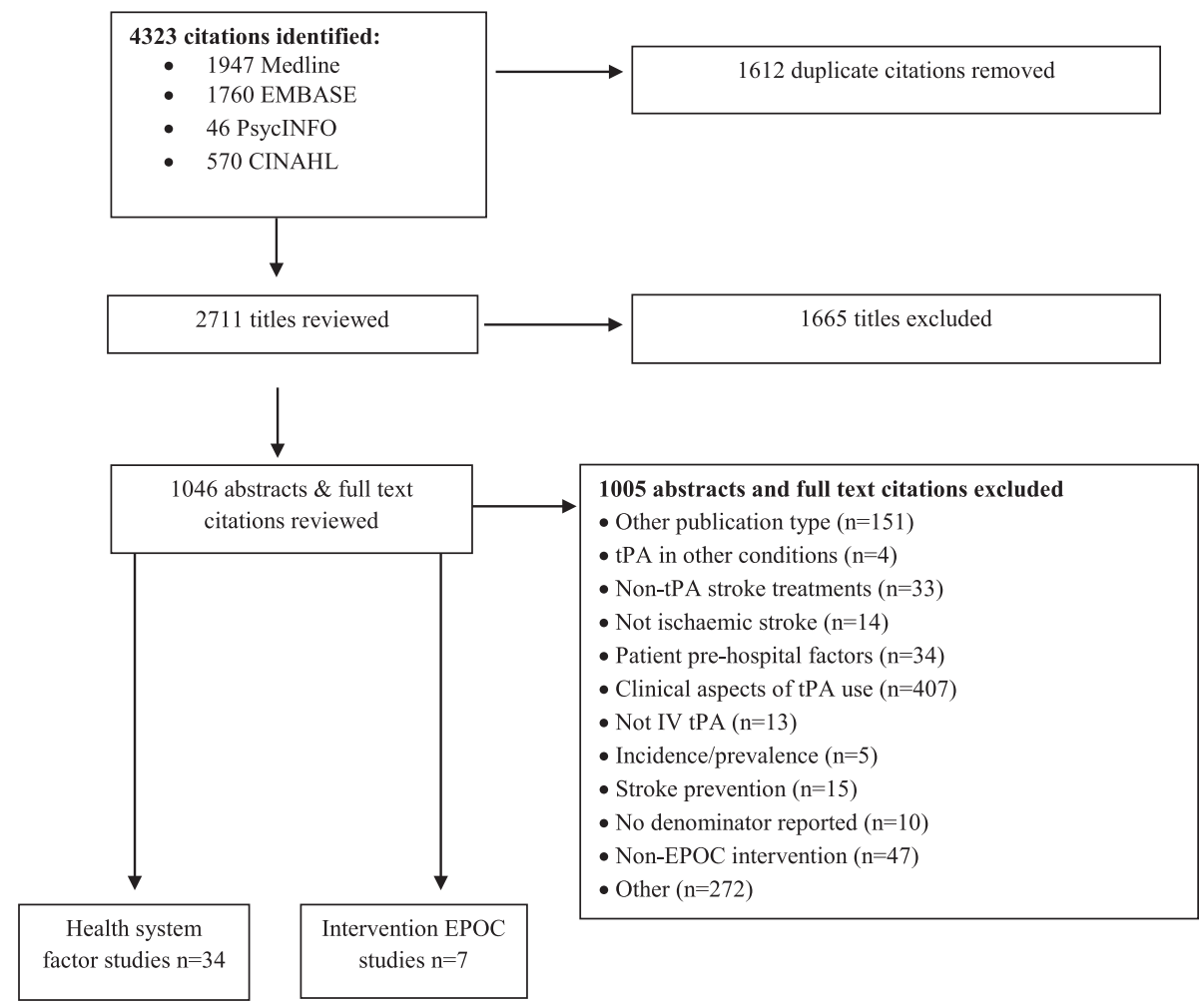

Fig. 1 Inclusion and exclusion of citations

\section{Health system factors}

Table 1 summarises the 34 studies exploring associations between thrombolysis rates and health system factors. The majority $(n=19)$ of studies conducted multivariable analyses including both health system and patient factors. Health system factors were categorised post hoc and those with predominantly positive associations with tPA rates were as follows:

- Travel time and location: e.g. urban rather than rural location, or a centralised/"hub" model linking outlying centres with other, generally larger, centres (environmental restructuring)

- Training, skills and expertise: treatment by neurologist or in a neurology department; admission to a stroke unit; treatment at academic/teaching hospital; treatment at a hospital with higher volume of stroke admissions or neurology beds; or accreditation as a "medical centre" (training and education)

- Facilities and staffing: having a neurologist, stroke nurse or stroke team; neurological or neuroimaging services; and weekend arrival (service provision)

- Organisational elements: use of stroke-specific protocols or transfer by ambulance/mobile emergency team rather than other means (guidelines and regulation)
The terms in parentheses refer to $\mathrm{BCW}$ intervention functions and policy categories.

\section{Effectiveness of system-focussed interventions}

Two intervention studies [38, 39] compared telemedicine with a telephone-only approach under the "hub and spoke" model. This group were too diverse in methodology and measurement to be included in a pooled analysis. Therefore, a narrative outcome description is provided. Neither of the telemedicine studies found a significant difference in IV tPA rates or patient outcomes, with one [38] aiming to assess feasibility rather than effectiveness resulting in limited power to find any effect. Meyer et al. [39] identified significantly higher rates of correct treatment decisions in telemedicinetreated patients compared to the telephone-only group. A third study [40] explored a hub and spoke teleconsultation approach for one group of sites while a control group of sites proceeded with usual care. All sites found significant increases in IV tPA, while only tele-consultation sites significantly reduced mortality.

Four studies [32, 41-43] explored approaches using quality improvement methods. Of these, two [41, 42] found a significant effect on IV tPA rates and patient outcomes based on modified Rankin scores. Scott et al. [43] found a significant effect on IV tPA rates for some analyses, with no significant effect on service delivery 
Table 1 Health system factors associated and not associated with higher thrombolysis rates

\begin{tabular}{|c|c|c|}
\hline Health system factors & $\begin{array}{l}\text { Studies finding no association } \\
\text { with higher thrombolysis rate }\end{array}$ & $\begin{array}{l}\text { Studies finding a significant association } \\
\text { with higher thrombolysis rate }\end{array}$ \\
\hline \multicolumn{3}{|l|}{ Travel time and location (environmental restructuring) ${ }^{a}$} \\
\hline Shorter transport time or distance to hospital & [48-51] & {$[52,53]$} \\
\hline Urban (vs rural) & - & [54-56] \\
\hline Centralised (hub model) & - & [57] \\
\hline \multicolumn{3}{|l|}{ Training, skills and expertise (training and education) ${ }^{a}$} \\
\hline Treated by a neurologist & - & {$[49,56],[58]$ (no statistical test) } \\
\hline Admitted to or treated in a neurology department or stroke unit & [59] & {$[60,61]$} \\
\hline Academic/teaching hospital & [56] & {$[55,60,62-64],[65]^{\mathrm{b}}$} \\
\hline Continuing medical education/formal stroke training & {$[33,62]$} & [25] \\
\hline Higher volume of stroke admissions/number of neuro beds & {$[56,59]$} & {$[49,61,66]$} \\
\hline Accreditation as medical centre & - & [49] \\
\hline \multicolumn{3}{|l|}{ Facilities and staffing (service provision) ${ }^{a}$} \\
\hline Emergency medical service or emergency department & [33] & [25] \\
\hline Neurologists, stroke nurse, stroke unit or team & [33] & {$[25,61,62,67]$} \\
\hline Neurological/neuroimaging services & [62] & {$[25,68]$} \\
\hline Laboratory services & {$[25,62]$} & - \\
\hline Larger/higher volume hospital & {$[56,61]$} & [69] \\
\hline Arrival during "on" hours & {$[57,70]$} & - \\
\hline Arrival on weekend & [70] & {$[49,71]$} \\
\hline $24 \mathrm{~h}$ or rapid CT/MRI & [62] & - \\
\hline Intensive care unit (cat 1) & [72] & - \\
\hline Stroke allocated beds & [33] & - \\
\hline \multicolumn{3}{|l|}{ Organisational elements (guidelines and regulations) ${ }^{a}$} \\
\hline Commitment of medical organisation or stroke centre director & [25] & [62] \\
\hline Quality improvement outcomes or activities & {$[25,62]$} & - \\
\hline Pre-hospital notifications or triage tool & {$[73,74]$} & [75] \\
\hline Stroke-related certification & [76] & [77] \\
\hline Ambulance agreements/protocols or training & [33] & [33] (borderline positive association) \\
\hline Who interprets $C T$ & [33] & - \\
\hline Stroke-specific protocols & [62] (acute stroke protocol) & {$[25,33,62]$} \\
\hline Transfer by a mobile emergency team or ambulance & - & {$[48,50,78,79]$} \\
\hline
\end{tabular}

${ }^{\mathrm{a}}$ Terms in parentheses refer to BCW intervention functions and policy categories

${ }^{\mathrm{b}}$ Significant in univariate analysis only

measures or patient outcome. Schwamm et al. [32] reported that involvement in the Get With The Guidelines Stroke programme was associated with an improvement over time in thrombolysis rates for patients arriving within $2 \mathrm{~h}$ of symptom onset.

The four quality improvement studies were included in pooled analysis of tPA rates. As the heterogeneity of the studies was high $\left(I^{2}=98 \%[95 \% \mathrm{CI}=97.1\right.$ to $98.5 \%]$ ), a random-effects model was used, and the pooled estimate should be treated with caution. A borderline significant effect was found, with a pooled odds ratio of $2.1(95 \% \mathrm{CI}=1.0$ to 4.5$)$ and; $X^{2}=3.783689, d f=1$, $p=0.05$. The seven intervention studies are described in Table 2.

\section{Discussion}

This systematic review brings together the empirical evidence regarding potential strategies for improving thrombolysis rates for acute stroke. The review data provide a basis on which stroke service providers can identify which strategies are more likely to be good investments for increasing rates of thrombolysis. As per the literature regarding complex interventions [14] and frameworks such as the $\mathrm{BCW}$ [20], a range of strategies 
Table 2 Intervention studies meeting EPOC criteria for study design $(n=7)$

\begin{tabular}{|c|c|c|c|c|c|c|c|c|}
\hline $\begin{array}{l}\text { Citation, trial name, } \\
\text { design, setting }\end{array}$ & $\begin{array}{l}\text { Target group, } \\
\text { study duration }\end{array}$ & $\begin{array}{l}\text { Randomization } \\
\text { methods }\end{array}$ & Eligibility & $\begin{array}{l}\text { ample size, response } \\
\text { te, representativeness }\end{array}$ & Intervention conditions & Outcome measures & Statistical analysis & Findings \\
\hline $\begin{array}{l}\text { Demaerschalk } \\
2010 \text { [38], USA } \\
\text { STRokE DOC AZ } \\
\text { RCT } \\
\text { Regional (spoke) } \\
\text { and Academic } \\
\text { Metropolitan } \\
\text { (hub) hospitals }\end{array}$ & $\begin{array}{l}\text { Hospital staff } \\
\text { Dec. 2007- } \\
\text { Oct. } 2008\end{array}$ & $\begin{array}{l}\text { Unit of analysis: } \\
\text { patient } \\
\text { Concealed } \\
\text { allocation: yes } \\
\text { Blinded: no } \\
\text { Allocation to } \\
\text { condition: } \\
\text { permuted block } \\
\text { randomization of } \\
\text { patients stratified } \\
\text { by site }\end{array}$ & $\begin{array}{l}\text { Patient: }>18 \\
\text { years } \\
\text { tPA window: } \\
\text { onset }<3 \mathrm{~h} \text {. }\end{array}$ & $\begin{array}{l}\text { Patient: } n=54 \\
\text { Hospital: } n=3 \\
\text { Response rate, } 68.4 \% \text {. } \\
\text { Representativeness: } \\
\text { no demographic } \\
\text { differences between } \\
\text { groups } \\
\text { Myocardial infarction } \\
\text { higher in int. group } \\
(p<0.02) \text {. }\end{array}$ & $\begin{array}{l}\text { Int-1: audio and } \\
\text { video contact with } \\
\text { a certified stroke } \\
\text { team at a hub site, } \\
\text { who had access } \\
\text { to medical history, } \\
\text { performed NIHSS, } \\
\text { and reviewed test } \\
\text { results and CT } \\
\text { images } \\
\text { Int-2: a hub stroke } \\
\text { consultant queried } \\
\text { history, physical exam } \\
\text { (including NIHSS), test } \\
\text { results, CT report }\end{array}$ & $\begin{array}{l}\text { tPA rate: } \\
\text { denominator }=\text { acute } \\
\text { stroke with }<3 \mathrm{~h} \\
\text { onset. } \\
\text { Service delivery: } \\
\text { 1. Evaluation times } \\
\text { (e.g. door-ED) } \\
\text { 2. Correct treatment } \\
\text { decision } \\
\text { Patient outcomes: } \\
\text { 1. Barthel Index } \\
\text { (score } 95-100) \\
\text { 2. mRS (score } \leq 2) \text {. }\end{array}$ & $\begin{array}{l}\text { Cochran-Mantel-Haenszel } \\
\text { test: comparison of } \\
\text { correct decision rate } \\
\text { between groups } \\
\text { Fisher's exact test: rate } \\
\text { of tPA, rate of intracranial } \\
\text { haemorrhage, mortality, } \\
90 \text { day mRS } \\
\text { Wilcoxon rank sum test: } \\
90 \text {-day Barthel Index } \\
\text { and time comparisons }\end{array}$ & $\begin{array}{l}\text { tPA rate: Int-1, } \\
30 \% \text {; Int-2, } 30 \% \\
\text { Service delivery: } \\
\text { 1. NS } \\
\text { 2. NS } \\
\text { Patient outcome: } \\
\text { 1. NS } \\
\text { 2. NS } \\
\text { Note: insufficient } \\
\text { power to assess } \\
\text { difference in tPA } \\
\text { rates between } \\
\text { groups. }\end{array}$ \\
\hline $\begin{array}{l}\text { Dirks, } 2011 \text { [41], } \\
\text { The Netherlands. } \\
\text { PRACTISE } \\
\text { Cluster RCT } \\
\text { Hospitals }\end{array}$ & $\begin{array}{l}\text { Hospital staff, } \\
\text { including } \\
\text { stroke } \\
\text { neurologist } \\
\text { and stroke } \\
\text { nurse } \\
\text { May 2005- } \\
\text { Jan. } 2008\end{array}$ & $\begin{array}{l}\text { Unit of analysis: } \\
\text { hospital } \\
\text { Concealed } \\
\text { allocation: no } \\
\text { Blinded: no } \\
\text { Allocation to } \\
\text { condition: hospitals } \\
\text { randomised after } \\
\text { pairwise matching } \\
\text { on hospital type, } \\
\text { tPA rate, stroke } \\
\text { patients/year }\end{array}$ & $\begin{array}{l}\text { Patient: }>18 \\
\text { years } \\
\text { Hospital: } \\
\text { 100-500 stroke } \\
\text { admissions/year } \\
\text { tPA window: } \\
<4 \text { h of onset }\end{array}$ & $\begin{array}{l}\text { Patient: } n=1657 \\
\text { Hospital: } n=12 \text {. } \\
\text { Response rate: } \\
\text { Not reported. } \\
\text { Representativeness: } \\
\text { patients: mean age, } \\
\text { sex distribution and } \\
\text { mean NIHSS at } \\
\text { admission were similar } \\
\text { between groups }\end{array}$ & $\begin{array}{l}\text { Int: } 5 \times \text { half day } \\
\text { (across } 2 \text { years) } \\
\text { meetings based on } \\
\text { Breakthrough Series } \\
\text { model. Teams of } \\
\text { stroke neurologist } \\
\text { and stroke nurse } \\
\text { were created, who } \\
\text { noted barriers to } \\
\text { tPA use, set goals } \\
\text { and plan actions } \\
\text { C: usual practices. }\end{array}$ & $\begin{array}{l}\text { tPA rate: } \\
\text { denominator }= \\
\text { ischemic stroke, } \\
<4 \text { h onset } \\
\text { Service delivery: } \\
\text { 1. Onset-to-door } \\
\text { time (min) } \\
\text { 2. Door-to-needle } \\
\text { time (min) } \\
\text { Patient outcome: } \\
\text { 1. mRS }<3 \text { (at } 3 \text { months) } \\
\text { 2. Quality of life-EuroQoL } \\
\text { (at } 3 \text { months) } \\
\text { 3. Mortality }\end{array}$ & $\begin{array}{l}\text { Intention to treat } \\
\text { Multilevel logistic } \\
\text { and linear regressions: } \\
\text { comparison of tPA use, } \\
\text { mRS, QoL and mortality } \\
\text { between intervention } \\
\text { groups. } \\
\text { Service delivery time } \\
\text { analysis was adjusted } \\
\text { for size, type and } \\
\text { previous tPA rates, } \\
\text { age, sex. }\end{array}$ & $\begin{array}{l}\text { tPA rate: Int, } 44 \% \text {; } \\
\text { C, } 39 \% \text { (unadjusted } \\
\text { OR = } 1.24 \text { [1.02-1.51]). } \\
\text { Service delivery: } \\
\text { 1. NS } \\
\text { 2. NS } \\
\text { Patient outcome: } \\
\text { 1. Poorer in C group } \\
\text { 2. NS } \\
\text { 3. NS }\end{array}$ \\
\hline $\begin{array}{l}\text { Meyer } 2008 \text { [39], } \\
\text { USA } \\
\text { STRokE DOC } \\
\text { RCT } \\
\text { Remote "spoke" } \\
\text { hospitals }\end{array}$ & $\begin{array}{l}\text { Hospital staff } \\
\text { Jan. 2004- } \\
\text { Aug. } 2007\end{array}$ & $\begin{array}{l}\text { Unit of analysis: } \\
\text { patient } \\
\text { Concealed } \\
\text { allocation: no } \\
\text { Blinded: no } \\
\text { Allocation to } \\
\text { condition: patients } \\
\text { randomised within } \\
\text { permuted blocks } \\
\text { stratified by site }\end{array}$ & $\begin{array}{l}\text { Patient: >18 } \\
\text { years and } \\
\text { ability to sign } \\
\text { consent } \\
\text { tPA window: } \\
<3 \mathrm{~h} \text { for } \\
\text { treatment, but } \\
\text { no time limit } \\
\text { on eligibility } \\
\text { for trial }\end{array}$ & $\begin{array}{l}\text { Patient: } n=222 \\
\text { (111 vs } 111) \\
\text { Hospital: } n=4 \\
\text { Response rate: } \\
\text { Patients: Not reported. } \\
\text { Representativeness: } \\
\text { No demographic } \\
\text { differences between } \\
\text { groups. Int-1 had higher } \\
\text { NIHSS score at } \\
\text { presentation than } \\
\text { Int- } 2(p<0.005) \text {. }\end{array}$ & $\begin{array}{l}\text { Int-1: telemedicine } \\
\text { (including video) } \\
\text { consultation with } \\
\text { patient by hub } \\
\text { consultant including } \\
\text { CT imaging } \\
\text { Int-2: telephone } \\
\text { consultations for } \\
\text { spoke sites with } \\
\text { hub consultants } \\
\text { Hub provided } \\
\text { treatment } \\
\text { recommendations } \\
\text { for both groups }\end{array}$ & $\begin{array}{l}\text { tPA rate: } \\
\text { denominator = acute } \\
\text { stroke. } \\
\text { Service delivery: } \\
\text { 1. Correct treatment } \\
\text { decisions } \\
\text { 2. Stroke onset to each } \\
\text { point of care pathway } \\
\text { (min) } \\
\text { Patient outcome: } \\
\text { 1. Barthel Index } \\
\text { (score } 95-100) \text {. } \\
\text { 2. mRS (score } \leq 2) \text {. }\end{array}$ & $\begin{array}{l}\text { Fisher's exact test: } \\
\text { difference in tPA rate, } \\
\text { functional outcomes }\end{array}$ & $\begin{array}{l}\text { tPA rate: Int-1, } \\
28 \% ; \text { Int-2, } 23 \% \\
\text { (OR }=1.3[0.7-2.5] \text {, } \\
p=\text { NS). } \\
\text { Service delivery: } \\
\text { 1. Greater in Int-1 } \\
\text { compared to } \\
\text { Int-2 ( } 98 \text { vs } 82 \% \text {, } \\
\mathrm{OR}=10.9[2.7-44.6] \text {, } \\
p<0.001 \text { ). } \\
\text { 2. Few differences in } \\
\text { service delivery times. } \\
\text { Patient outcome: } \\
\text { 1. No difference } \\
\text { between groups } \\
\text { 2. No difference } \\
\text { between groups }\end{array}$ \\
\hline
\end{tabular}


Table 2 Intervention studies meeting EPOC criteria for study design $(n=7)$ (Continued)

\begin{tabular}{|c|c|c|c|c|c|c|c|c|}
\hline $\begin{array}{l}\text { Morgenstern et al. } \\
2003 \text { [42], USA } \\
\text { TTL Temple } \\
\text { Foundation } \\
\text { Stroke Project } \\
\text { CBA } \\
\text { Hospitals in } \\
\text { two communities }\end{array}$ & $\begin{array}{l}\text { Community } \\
\text { members } \\
\text { and hospital } \\
\text { staff } \\
\text { Feb. 1998- } \\
\text { Sept. } 2000\end{array}$ & $\begin{array}{l}\text { Unit of analysis: } \\
\text { patient } \\
\text { Concealed } \\
\text { allocation: no } \\
\text { Blinded: no } \\
\text { Allocation to } \\
\text { condition: } \\
\text { comparison } \\
\text { community selected } \\
\text { to match chosen } \\
\text { intervention } \\
\text { community }\end{array}$ & $\begin{array}{l}\text { Patient: }>21 \\
\text { years and } \\
\text { county } \\
\text { resident } \\
\text { tPA window: } \\
<3 \mathrm{~h}\end{array}$ & $\begin{array}{l}\text { Patient: } \\
\text { Phase 1: } n=277 \\
\text { (136 vs 141) } \\
\text { Phase 2: } n=499 \\
\text { (266 vs 233) } \\
\text { Phase 3: } n=150 \\
\text { (80 vs 70) } \\
\text { Hospital: } n=10 \\
\text { Response rate: } \\
\text { Patients: N/A } \\
\text { Hospitals: not reported } \\
\text { Representativeness: } \\
\text { hospital characteristics } \\
\text { reported }\end{array}$ & $\begin{array}{l}\text { Int: community } \\
\text { mass media, } \\
\text { hospital-based } \\
\text { systems change } \\
\text { via multi-disciplinary } \\
\text { team development } \\
\text { of ED protocols, } \\
\text { problem solving, } \\
\text { medical education, } \\
\text { feedback. } \\
\text { C: not specified. }\end{array}$ & $\begin{array}{l}\text { tPA rate: } \\
\text { denominator = } \\
\text { ischemic } \\
\text { stroke } \\
\text { Service delivery: } \\
\text { 1. Delay time to } \\
\quad \text { hospital } \\
\text { 2. Staff-reported } \\
\text { barriers to } \\
\text { treatment } \\
\text { Patient outcome: } \\
\text { none assessed }\end{array}$ & $\begin{array}{l}\text { Fisher's exact test: } \\
\text { rate of tPA } \\
\text { ANOVA: delay } \\
\text { in times }\end{array}$ & $\begin{array}{l}\text { tPA rate: Int } \\
\text { (phases 1-3): } 2.2, \\
8.6,11.2 \%(p<0.007) ; \\
\text { C (phases } 1-3): 0.7, \\
0.9 \%,(p=\text { NS) } \\
\text { Service delivery: } \\
\text { 1. No difference in } \\
\text { either group } \\
\text { 2. Reduction for Int } \\
\quad \text { group only (no } \\
\text { statistical test) }\end{array}$ \\
\hline $\begin{array}{l}\text { Schwamm et al. } \\
2009 \text { [32], USA } \\
\text { ITS } \\
\text { Academic and } \\
\text { community } \\
\text { hospitals }\end{array}$ & $\begin{array}{l}\text { Hospitals } \\
\text { April 2003- } \\
\text { July } 2007\end{array}$ & $\begin{array}{l}\text { Unit of analysis: } \\
\text { hospital } \\
\text { Concealed } \\
\text { allocation: N/A } \\
\text { Blinded: N/A } \\
\text { Allocation to } \\
\text { condition: N/A } \\
\text { (ITS design) }\end{array}$ & $\begin{array}{l}\text { Patient: } \\
\text { Principal } \\
\text { diagnosis of } \\
\text { stroke or TIA, } \\
\text { arrival }<2 \mathrm{~h} \\
\text { from onset, } \\
\text { ICD-9. Retro } \\
\text { spective chart } \\
\text { review to } \\
\text { confirm } \\
\text { stroke/TIA } \\
\text { Hospital: }>30 \\
\text { patients }\end{array}$ & $\begin{array}{l}\text { Patient: } \\
n=322,847 \\
\text { (ischemic }=73.2 \% ; \\
\text { TIA }=26.8 \% \text { ) } \\
\text { Hospital: } n=790 \\
\text { Response rate: } \\
\text { Unclear. Staggered } \\
\text { recruitment over } \\
4 \text { years. By Jan. } \\
2007,8.35 \% \text { hospitals } \\
\text { had dropped out } \\
(n=66) \\
\text { Representativeness: } \\
\text { hospital characteristics } \\
\text { provided }\end{array}$ & $\begin{array}{l}\text { Int: quality } \\
\text { improvement } \\
\text { (Get With The } \\
\text { Guidelines } \\
\text { [GWTG]) programme, } \\
\text { with organisational } \\
\text { meetings, tool } \\
\text { kits, collaborative } \\
\text { workshops, hospital } \\
\text { recognition, decision } \\
\text { support information, } \\
\text { performance } \\
\text { feedback. }\end{array}$ & $\begin{array}{l}\text { tPA rate: } \\
\text { denominator = } \\
\text { stroke or TIA, } \\
\text { and arrival }<2 \mathrm{~h} \\
\text { of onset } \\
\text { Service delivery: } \\
\text { none assessed } \\
\text { Patient outcome: } \\
\text { 1. Symptomatic } \\
\text { intracranial } \\
\text { haemorrhage } \\
\text { within } 36 \mathrm{~h} \text { of tPA }\end{array}$ & $\begin{array}{l}\text { Cochran-Mantel- } \\
\text { Haenszel test: mean } \\
\text { score for changes } \\
\text { in rate of tPA } \\
\text { and intracranial } \\
\text { haemorrhage } \\
\text { over time }\end{array}$ & $\begin{array}{l}\text { tPA rate: significant } \\
\text { increase from } \\
\text { baseline }(42.1 \%) \text { to } \\
\text { year } 5(72.8 \% \text {; } \\
p<0.0001) \text {. } \\
\text { Patient outcome: } \\
\text { 1. NS over time } \\
\text { Greatest improvement } \\
\text { (composite performance/ } \\
\text { program year in GWTG) } \\
\text { in hospitals with more } \\
\text { beds }(p<0.0001) \text {, larger } \\
\text { annual stroke volume } \\
\text { ( } p<0.0001) \text { and teaching } \\
\text { status }(p<0.0001)\end{array}$ \\
\hline $\begin{array}{l}\text { Scott et al. } 2013 \\
\text { [43], USA } \\
\text { INSTINCT } \\
\text { Cluster RCT } \\
\text { Community } \\
\text { hospitals }\end{array}$ & $\begin{array}{l}\text { Physicians, } \\
\text { pharmacists, } \\
\text { nurses, EMS, } \\
\text { admin teams } \\
\text { Jan.-Dec. } 2007\end{array}$ & $\begin{array}{l}\text { Unit of analysis: } \\
\text { hospital } \\
\text { Concealed } \\
\text { allocation: no } \\
\text { Blinded: no } \\
\text { Allocation to } \\
\text { condition: within } \\
\text { pairs, hospitals were } \\
\text { randomised to } \\
\text { intervention or } \\
\text { control groups. } \\
\text { Randomisation } \\
\text { reversed for three } \\
\text { pairs to achieve } \\
\text { greater urban/rural } \\
\text { balance }\end{array}$ & $\begin{array}{l}\text { Hospitals: } \\
\text { discharging } \\
\geq 100 \text { stroke } \\
\text { patient/year, } \\
<100000 \text { ED } \\
\text { visits/year and } \\
\text { non-academic } \\
\text { stroke centres } \\
\text { tPA window: } \\
\text { not specified }\end{array}$ & $\begin{array}{l}\text { Hospitals: } \\
n=24 \\
\text { Response rate: } 83 \% \\
\text { Representativeness: } \\
\text { not reported }\end{array}$ & $\begin{array}{l}\text { Int: clinical practice } \\
\text { guideline promotion, } \\
\text { development of local } \\
\text { stroke champions, } \\
\text { continuing education, } \\
\text { telephone support } \\
\text { for treatment decision, } \\
\text { academic detailing, } \\
\text { audit and feedback } \\
\text { c: usual practices }\end{array}$ & $\begin{array}{l}\text { tPA rate: } \\
\text { denominator = } \\
\text { ischemic stroke } \\
\text { Service delivery: } \\
\text { 1. Adherence } \\
\text { to tPA guidelines } \\
\text { Patient outcome: } \\
\text { 1. Safety data } \\
\text { from proportion } \\
\text { of patients ( } 2.2 \%) \text {, } \\
\text { with reported } \\
\text { haemorrhage }\end{array}$ & $\begin{array}{l}\text { Intention-to-treat (ITT) } \\
\text { and target population } \\
\text { (without one pair that } \\
\text { was excluded after } \\
\text { randomisation) } \\
\text { Generalised linear } \\
\text { mixed model: assumed } \\
\text { intra-hospital correlation } \\
\text { between tPA rates at } \\
\text { pre- and post- } \\
\text { intervention periods }\end{array}$ & $\begin{array}{l}\text { tPA rate: ITT: Int } \\
\text { (pre and post), } 1.25 \\
\text { and } 2.79 \% ; C(n=1 ; \\
\text { pre and post), } 1.25 \\
\text { and } 2.10 \% \text {. Int vs } C \text {, } \\
p=\text { NS. } \\
\text { Target analysis: } \\
\text { Int (pre and post), } 1.0 \\
\text { and } 2.62 \% ; C \text { (pre and } \\
\text { post), } 1.09 \text { and } 1.72 \% \text {. } \\
\text { Int v } C, R R=1.68 \\
\text { [1.09-2.57], } p=0.02 \\
\text { Service delivery: } \\
\text { 1. NS difference } \\
\text { between groups } \\
\text { Patient outcome: } \\
\text { 1. NS difference } \\
\text { between groups }\end{array}$ \\
\hline
\end{tabular}


Table 2 Intervention studies meeting EPOC criteria for study design ( $n=7$ ) (Continued)

\begin{tabular}{|c|c|c|c|c|c|c|c|c|}
\hline $\begin{array}{l}\text { Theiss et al. } \\
2013 \text { [40], } \\
\text { Germany } \\
\text { CBA } \\
\text { Comprehensive } \\
\text { stroke centres, } \\
\text { and primary } \\
\text { care hospitals }\end{array}$ & $\begin{array}{l}\text { Hospitals } \\
\text { 2006-2009 }\end{array}$ & $\begin{array}{l}\text { Unit of analysis: } \\
\text { hospital } \\
\text { Concealed } \\
\text { allocation: no } \\
\text { Blinded: not } \\
\text { reported } \\
\text { Allocation to } \\
\text { condition: hospitals } \\
\text { matched on beds, } \\
\text { distance from } \\
\text { closest hub site } \\
\text { and departments } \\
\text { of internal medicine }\end{array}$ & $\begin{array}{l}\text { Hospitals: not } \\
\text { reported } \\
\text { No study } \\
\text { hospitals had } \\
\text { specialised } \\
\text { stroke care } \\
\text { prior to } \\
\text { study start }\end{array}$ & $\begin{array}{l}\text { Hospitals: } n=15 \\
\text { Response rate: not } \\
\text { reported. } \\
\text { Representativeness: } \\
\text { not reported }\end{array}$ & $\begin{array}{l}\text { Int: tele-consultation } \\
\text { service. Consisted of } \\
\text { hub }(n=5) \text { and } \\
\text { spoke }(n=5) \text { sites } \\
\text { C: usual practices }\end{array}$ & $\begin{array}{l}\text { tPA rate } \\
\text { denominator: all } \\
\text { stroke } \\
\text { Service delivery: } \\
\text { none assessed } \\
\text { Patient outcome: } \\
\text { 1. Intracerebral } \\
\text { haemorrhage } \\
\text { 2. Mortality }\end{array}$ & $\begin{array}{l}\text { Mean and SEM: for } \\
\text { descriptive data } \\
\text { Student } t \text { and Fisher } \\
\text { exact tests: longitudinal } \\
\text { and pairwise comparisons, } \\
\text { pooled ischemic } \\
\text { stroke mortality }\end{array}$ & $\begin{array}{l}\text { tPA rate: Hub sites: } \\
\text { (pooled) increased } 4.2 \\
\text { to } 7.7 \%(p<0.0001) ; \\
\text { Spoke sites: (pooled) } \\
\text { increased } 1.1 \text { to } 5.9 \% \\
\text { ( } p<0.0001) ; C: \\
\text { (one hospital only) } \\
\text { increased } 0.8 \text { to } 5.7 \% \\
\text { ( } p=0.03 \text { ). } \\
\text { Patient outcome: } \\
\text { 1. NS } \\
\text { 2. Significant } \\
\text { decreases in spoke } \\
\text { site only ( } 10.3 \text { to } \\
7.3 \%, p=0.03)\end{array}$ \\
\hline
\end{tabular}

Abbreviations: $C$ control group, CBA controlled before and after trial, $C T$ computer tomography, ED emergency department, EMS emergency medical service, $R C T$ randomised controlled trial, Int intervention group, ITS interrupted time series, mRS modified Rankin score, NIHSS National institute of Health Stroke Scale, TIA transient ischemic attack, $t P A$ tissue plasminogen activator, QoL quality of life, N/A not applicable 
or factors are related to achieving change in thrombolysis rates. Of note is that the literature only addresses three of the nine intervention functions and two of the seven policy categories raised in the BCW framework, suggesting a much wider range of strategies could be tested in the future.

A small number of system-related factors are associated with higher rates of IV TPA administration for ischemic stroke. Systems-change interventions, based on multi-component quality improvement approaches, can increase the proportion of eligible stroke patients receiving IV tPA.

The observational literature regarding factors associated with higher stroke tPA rates was heterogeneous in methodology and types of factors assessed, but it is unclear whether each study had sufficient power to detect an association for each factor. The literature indicates that health systems should aim to ensure that most stroke patients are treated in a way that minimises access disadvantages for rural populations; maximises access to neurological and stroke-specific expertise and experience; ensures stroke units are widely available; and implements stroke-specific protocols. The association of higher IV tPA rates with treatment at a teaching hospital or a hospital with larger stroke or IV tPA treatment volume suggests that expertise and experience within such settings is key to increased IV tPA rates. The mixed findings regarding the importance of treatment at a larger hospital and arrival during "on" hours or weekends indicate that greater size and availability of staff alone do not produce higher IV tPA rates. However, it must be noted that observational studies cannot be used to draw definitive conclusions regarding causation. The observational studies were also largely retrospective in design and had limited capacity to identify and assess confounding factors. Therefore, a greater focus must be directed towards the data from the experimental or intervention studies.

Organisational elements such as stroke certification and quality improvement activities were not associated with higher IV tPA rates. One study [25] failed to find an association between facilities, staffing and organisational elements and quality improvement outcomes or activities. These elements are often the focus of systemchange interventions and can be resource intensive to implement. Therefore, robust experimental studies are essential to providing clarity about cost-effective approaches to improved IV tPA rates. Organisational elements such as stroke-related certification or time on the Get With The Guidelines programme did not increase IV tPA rates.

The intervention studies suggest that while quality-improvement or system-change interventions can be effective in increasing IV tPA rates, studies are heterogeneous and effects may be small or inconsistent. The PRACTISE trial [41] found a positive effect on IV tPA rates and patient functioning following a Breakthrough Series intervention. The INSTINCT trial [43] reported a positive effect only when the analysis focussed on a subset of study sites. The INSTINCT intervention placed less emphasis on collaborative meetings compared to the PRACTISE trial but included stroke champions, education/support for treatment decision making and performance feedback [43]. The Morgenstern et al. study [42] identified a greater increase in IV tPA rates in intervention sites, compared to control sites.

This study [42] differed from the two other studies by including community-focussed mass media. It also included hospital-based change via multi-disciplinary teams, development of emergency department protocols, problem solving, medical education and performance feedback. Given the small number of hospitals involved, the choice of patient rather than hospital as the unit of analysis, and the lack of any head-to-head analysis across groups, some caution should be applied to interpreting the results of the Morgenstern et al. study [42].

Other studies support the finding that quality improvement strategies can provide modest positive effects on other aspects of stroke care [32, 44, 45]. The "Stroke 90:10" trial found an $11 \%$ relative improvement in some aspects of initial assessment and care for stroke patients following collaborative quality improvement [45]. Another study involving workshops, education, site-based teams, performance feedback and decisional support suggests that improvements in thrombolysis occurred over time [32]. While the study could be classified as an interrupted time series based on quarterly measurements over 4 years, the analysis did not follow usual approaches to analysing time-series data. Although the cost of IV tPA administration was not addressed in the reviewed studies, the scope of multi-component, multisite interventions suggests the resources required are substantial.

Two studies [38, 39] compared telemedicine with a telephone-only approach, focusing on environmental restructuring rather than quality improvement to increase IV tPA delivery. Conclusions are difficult to make, as neither study indicated sufficient power to detect a difference in IV tPA rates. The study of tele-consultation compared to usual care [40] suggested, but did not conclusively demonstrate, patient outcome benefits, given a failure to statistically examine experimental versus control site outcomes. A later pooled analysis confirmed telemedicine consultations were not associated with increased thrombolysis rates [46]. While these changes may increase access to expert care, they lack robust evidence. 
Observational and intervention data suggest that optimising IV tPA administration requires availability of expertise and protocols. Intervention studies suggest more in-depth reporting of the degree to which various intervention strategies may assist in understanding the best way forward. While multi-component approaches appear promising, two important questions emerge:

- Could a comprehensive intervention approach, encompassing the range of strategies represented in the reviewed studies, achieve a more substantial increase in IV tPA rates than that found to date? If so, what is the cost-benefit?

- Could a more streamlined quality improvement approach be identified, using a subset of elements? This may require comprehensive and systematic approaches to study the implementation of prior and future multi-component interventions, followed by trials using a subset of "best-bet" strategies.

The broader context is also important to consider, such as the financial incentives to hospitals for or against thrombolysis delivery in certain settings [21]. Adapting system thinking where components of the health system are dynamic and interlinked may assist in further understanding the network of relations and feedback loops impacting on the uptake of new innovations [47]. It may also be useful to develop a broader theoretical framework that could be applied to future studies in this area.

Limitations should be considered when interpreting the study tables: firstly, the reported rates of IV tPA (see Table 2) are dependent on denominator and eligibility criteria, which can affect the power of the study to detect a difference in the outcome; secondly, the variability in factors explored across studies of descriptive health system factors limits the ability to make comparisons among studies; and finally, the nature of changing care at a system level limits design rigour such as the ability to blind sites to group allocation. As a "Google" search was not used, a small number of unpublished studies may not have been identified.

\section{Conclusion}

Access to teaching hospitals and hospitals with larger stroke and IV tPA treatment volumes is associated with increased IV tPA administration rates for stroke, although results should be viewed against variability in eligibility criteria and type of denominator used. Interventions aiming to increase rates of IV tPA are resource intensive and comparisons between studies are difficult due to insufficient power and limitations in study analysis. More empirical data regarding the effects of efforts to improve access to thrombolysis for those living long distances (e.g. mobile thrombolysis) from a tPA-capable hospital and 24-h availability of expertise in acute stroke care are required, as is more thoughtfully designed and well-reported trials of quality improvement interventions.

\section{Competing interests}

The authors declare that they have no competing interests.

\section{Authors' contributions}

All authors contributed to the conceptualization of the manuscript. CP, AR, $\mathrm{SR}, \mathrm{CK}, \mathrm{JA}$ and EK were involved in the searching and data extraction process. AR, CP and SR drafted the manuscript. All authors read, provided critical review and approved the final manuscript.

\section{Acknowledgements}

We would like to thank Ms. Debbie Booth, The University of Newcastle medical librarian, for assistance with search strategy and terminology. This work is related to a National Health and Medical Research Council (NHMRC) partnership grant (ID569328) and is part-funded by a TRIP fellowship, with collaborative funding from Boehringer Ingelheim and in-kind support from ACI Stroke Care Network/Stroke Services NSW, Victorian Stroke Clinical Network, National Stroke Foundation and NSW Cardiovascular Research Network-National Heart Foundation, with infrastructure funding from Hunter Medical Research Institute and The University of Newcastle. Christine Paul is supported by an NHMRC Career Development Fellowship (APP1061335). None of these funding sources have had any involvement whatsoever with regard to the concept, development, writing and publishing of this paper.

\section{Author details}

${ }^{1}$ The University of Newcastle, University Drive, Callaghan, NSW 2308, Australia. ${ }^{2}$ Hunter New England Health, Lookout Road, New Lambton Heights, NSW 2305, Australia. ${ }^{3}$ Hunter Medical Research Institute, 1/Kookaburra Circuit, New Lambton Heights, NSW 2305, Australia.

Received: 24 June 2015 Accepted: 28 March 2016

Published online: 08 April 2016

\section{References}

1. Lopez AD, Mathers CD, Ezatti M, Jamieson DT, Murray DJL. Global burden of disease and risk factors. New York/Washington: Oxford University Press and the World Bank; 2006

2. Mackay J, Mensah G. The atlas of heart disease and stroke. Geneva: WHO; 2004.

3. Agency for Healthcare Research and Quality. Household component summary table. In: Medical Expenditure Panel Survey. U.S. Department of Health and Human Services. 2012. http://meps.ahrq.gov/mepsweb/. Accessed 12 Nov 2012.

4. Australian Bureau of Statistics. Cardiovascular disease in Australia: a snapshot, 2004-05. Canberra: ABS; 2006.

5. Australian Institute of Health and Welfare. Australia's health 2006. Report. Canberra: AlHW; 2006. Report No.:1032-6138.

6. Deloitte Access Economics. The economic impact of stroke in Australia. Melbourne: NSF; 2013.

7. National Stroke Foundation. National stroke audit-acute services clinical audit report 2011. Report. Melbourne: NSF; 2011.

8. Hankey GJ, Jamrozik K, Broadhurst RJ, Forbes S, Anderson CS. Long-term disability after first-ever stroke and related prognostic factors in the Perth Community Stroke Study, 1989-1990. Stroke. 2002;33(4):1034-40.

9. Saver J, Fonarow G, Smith E, Reeves M, Grau-Sepulveda M, Pan W, et al. Time to treatment with intravenous tissue plasminogen activator and outcome from acute ischemic stroke. JAMA. 2013;309(23):2480-8.

10. Hacke W, Kaste M, Bluhmki E, Brozman M, Dávalos A, Guidetti D, et al. Thrombolysis with alteplase 3 to 4.5 hours after acute ischemic stroke. N Engl J Med. 2008;359(13):1317-29.

11. Ahmed N, Wahlgren N, Grond M, Hennerici M, Lees KR, Mikulik R, et al. Implementation and outcome of thrombolysis with alteplase 3-4.5 h after an acute stroke: an updated analysis from SITS-ISTR. Lancet Neurol. 2010;9(9):866-74.

12. Strbian $D$, Michel $P$, Ringleb $P$, Numminen $H$, Breuer $L$, Bodenant $M$, et al. Relationship between onset-to-door time and door-to-thrombolysis time: a pooled analysis of 10 dedicated stroke centers. Stroke. 2013;44(10):2808-13. 
13. Quain DA, Parsons MW, Loudfoot AR, Spratt NJ, Evans MK, Russell ML, et al. Improving access to acute stroke therapies: a controlled trial of organised pre-hospital and emergency care. Med J Aust. 2008;189(8):429-33.

14. Craig P, Dieppe P, Macintyre S, Michie S, Nazareth I, Petticrew M. Developing and evaluating complex interventions: the new Medical Research Council guidance. BMJ. 2008:337.

15. Bunch ME, Nunziato EC, Labovitz DL. Barriers to the use of intravenous tissue plasminogen activator for in-hospital strokes. J Stroke Cerebrovasc Dis. 2012;21(8):808-11

16. Johnson M, Bakas T. A review of barriers to thrombolytic therapy: implications for nursing care in the emergency department. J Neurosci Nurs. 2010;42(2):88-94

17. Kwan J, Hand P, Sandercock P. A systematic review of barriers to delivery of thrombolysis for acute stroke. Age Ageing. 2004;33(2):116-21.

18. Upchurch GR, Dimick JB, Wainess RM, Eliason JL, Henke PK, Cowan JA, et al. Diffusion of new technology in health care: the case of aorto-iliac occlusive disease. Surgery. 2004;136(4):812-8.

19. Jaffe $A B$, Newell RG, Stavins RN. A tale of two market failures: technology and environmental policy. Ecol Econ. 2005;54(2):164-74.

20. Michie S, van Stralen MM, West R. The behaviour change wheel: a new method for characterising and designing behaviour change interventions. Implement Sci. 2011;6(1):42.

21. Adeoye $\mathrm{O}$, Hornung $\mathrm{R}$, Khatri $\mathrm{P}$, Kleindorfer D. Recombinant tissue-type plasminogen activator use for ischemic stroke in the United States: a doubling of treatment rates over the course of 5 years. Stroke. 2011;42(7):1952-5.

22. Bergman $M$, Ori $Y$, Blumberger $N$, Salman $H$. An alternative facility for a stroke unit in a community hospital. Eur J Intern Med. 2011;22(5):505-8.

23. Dick AP, Straka J. IV tPA for acute ischemic stroke: results of the first 101 patients in a community practice. Neurologist. 2005;11(5):305-8.

24. Slaght SJ, Weir NU, Lovett JK. The workload of stroke thrombolysis: a prospective study in a district general hospital setting. Acute Med. 2011;10(1):10-2.

25. Douglas VC, Tong DC, Gillum LA, Zhao S, Brass LM, Dostal J, et al. Do the brain attack coalition's criteria for stroke centers improve care for ischemic stroke? Neurology. 2005;64(3):422-7.

26. Prabhakaran S, McNulty M, O'Neill K, Ouyang B. Intravenous thrombolysis for stroke increases over time at primary stroke centers. Stroke. 2012;43(3):875-7.

27. Ranta A, Chan C, Rump D, Cariga P, Anderson L. Safety and efficacy of stroke thrombolysis at a secondary provincial hospital in New Zealand. N Z Med J. 2012;125(1358):35-43.

28. Salami S, Kolluru A, Al-Najafi S, Stover C, Mar A, Szpunar S, et al. Utilization and outcome of thrombolytic therapy for acute ischemic stroke: the St. John Hospital code stroke experience. J Clin Outcomes Manag. 2011;18(4):165-9.

29. Moynihan B, Davis D, Pereira A, Cloud G, Markus HS. Delivering regional thrombolysis via a hub-and-spoke model. J R Soc Med. 2010;103(9):363-9.

30. Nelson RE, Saltzman GM, Skalabrin EJ, Demaerschalk B, Majersik JJ. The cost-effectiveness of telestroke in the treatment of acute ischemic stroke. Neurology. 2011;77(17):1590-8.

31. Schwamm LH, Audebert HJ, Amarenco P, Chumbler NR, Frankel MR, George $M G$, et al. Recommendations for the implementation of telemedicine within stroke systems of care: a policy statement from the American Heart Association. Stroke. 2009;40:2635-60.

32. Schwamm LH, Fonarow GC, Reeves MJ, Pan W, Frankel MR, Smith EE, et al. Get With The Guidelines-Stroke is associated with sustained improvement in care for patients hospitalized with acute stroke or transient ischemic attack. Circulation. 2009;119(1):107-15.

33. van Wijngaarden JDH, Dirks M, Niessen LW, Huijsman R, Dippel DWJ. Do centres with well-developed protocols, training and infrastructure have higher rates of thrombolysis for acute ischemic stroke? QJM. 2011;104(9):785-91.

34. FDA. Alteplase product approval information-licensing action 6/18/96. 04/02/2009 ed: U.S. Food and Drug Administration; 1996.

35. Adams HP, Brott TG, Furlan AJ, Gomez CR, Grotta J, Helgason CM, et al. Guidelines for thrombolytic therapy for acute stroke: a supplement to the guidelines for the management of patients with acute ischemic stroke: a statement for healthcare professionals from a special writing group of the stroke council. Am Heart Assoc Circ. 1996;94(5):1167-74.

36. Popay J, Roberts H, Sowden A, Petticrew M, Arai L, Rodgers M, et al. Guidance on the conduct of narrative synthesis in systematic reviews, A product from the ESRC methods programme version. 2006. p. 1.
37. American Heart Association. Get With The Guidelines-Stroke. 2014 http://www.heart.org/HEARTORG/HealthcareResearch/GetWithThe Guidelines/Get-With-The-Guidelines-Stroke_UCM_306098_SubHomePage.jsp. Accessed 10 Jan 2014.

38. Demaerschalk BM, Bobrow BJ, Raman R, Kiernan T-EJ, Aguilar MI, Ingall TJ, et al. Stroke team remote evaluation using a digital observation camera in Arizona: the initial Mayo Clinic experience trial. Stroke. 2010;41(6):1251-8.

39. Meyer BC, Raman R, Hemmen T, Obler R, Zivin JA, Rao R, et al. Efficacy of site-independent telemedicine in the STRokE DOC trial: a randomised, blinded, prospective study. Lancet Neurol. 2008;7(9):787-95.

40. Theiss S, Günzel F, Storm A, Hausn P, Isenmann S, Klisch J, et al. Using routine data for quality assessment in NeuroNet telestroke care. J Stroke Cerebrovasc Dis. 2013;22(7):984-90.

41. Dirks M, Koudstaal PJ, Dippel DWJ, Niessen LW, van Wijngaarden JDH, Franke $\mathrm{CL}$, et al. Effectiveness of thrombolysis with intravenous alteplase for acute ischemic stroke in older adults. J Am Geriatr Soc. 2011;59(11):2169-71.

42. Morgenstern LB, Bartholomew LK, Grotta JC, Staub L, King M, Chan W. Sustained benefit of a community and professional intervention to increase acute stroke therapy. Arch Intern Med. 2003;163(18):2198-202.

43. Scott PA, Meurer WJ, Frederiksen SM, Kalbfleisch JD, Xu Z, Haan MN, et al. A multilevel intervention to increase community hospital use of alteplase for acute stroke (INSTINCT): a cluster-randomised controlled trial. Lancet Neurol. 2013;12(2):139-48.

44. Fonarow GC, Smith EE, Saver JL, Reeves MJ, Hernandez AF, Peterson ED, et al. Improving door-to-needle times in acute ischemic stroke: the design and rationale for the American Heart Association/American Stroke Association's target: stroke initiative. Stroke. 2011;42(10):2983-9.

45. Power M, Tyrrell PJ, Rudd AG, Tully MP, Dalton D, Marshall M, et al. Did a quality improvement collaborative make stroke care better? A cluster randomized trial. Implement Sci. 2014;9(40):9pp.

46. Demaerschalk BM, Raman R, Ernstrom K, Meyer BC. Efficacy of telemedicine for stroke: pooled analysis of the Stroke Team Remote Evaluation Using a Digital Observation Camera (STRokE DOC) and STRokE DOC Arizona telestroke trials. Telemed J E Health. 2012;18(3):230-7.

47. Atun R. Health systems, systems thinking and innovation. Health Policy Plan. 2012;27 suppl 4:iv4-8.

48. Kunisawa S, Morishima T, Ukawa N, Ikai H, Otsubo T, Ishikawa KB, et al. Association of geographical factors with administration of tissue plasminogen activator for acute ischemic stroke. J Am Heart Assoc. 2013;2(5), e000336.

49. Hsieh $\mathrm{CY}$, Chen $\mathrm{CH}$, Chen YC, Kao Yang YH. National survey of thrombolytic therapy for acute ischemic stroke in Taiwan 2003-2010. J Stroke Cerebrovasc Dis. 2013:22(8):e620-7.

50. Kunisawa S, Kobayashi D, Lee J, Otsubo T, Ikai H, Yokota C, et al. Factors associated with the administration of tissue plasminogen activator for acute ischemic stroke. J Stroke Cerebrovasc Dis. 2014;23(4):724-31.

51. Albright KC, Schott TC, Jafari N, Wohlford-Wessels MP, Finnerty EP, Jacoby MRK. Tissue plasminogen activator use: Evaluation and initial management of ischemic stroke from an lowa Hospital perspective. J Stroke Cerebrovasc Dis. 2005;14(3):127-35.

52. Leyden JM, Chong WK, Kleinig T, Lee A, Field JB, Jannes J. A populationbased study of thrombolysis for acute stroke in South Australia. Med J Aust. 2011;194(3):111-5.

53. Acharya AB, Nyirenda JC, Higgs GB, Bloomfield MS, Cruz-Flores S, Connor LT, et al. Distance from home to hospital and thrombolytic utilization for acute ischemic stroke. J Stroke Cerebrovasc Dis. 2011;20(4):295-301.

54. Kozera G, Chwojnicki K, Gojska-Grymajło A, Gasecki D, Schminke U, Nyka WM. Pre-hospital delays and intravenous thrombolysis in urban and rural areas. Acta Neurol Scand. 2012;126:171-7.

55. Nasr DM, Brinjikji W, Cloft HJ, Rabinstein AA. Utilization of intravenous thrombolysis is increasing in the United States. Int J Stroke. 2013;8(8):681-8.

56. Reed SD, Cramer SC, Blough DK, Meyer K, Jarvik JG. Treatment with tissue plasminogen activator and inpatient mortality rates for patients with ischemic stroke treated in community hospitals. Stroke. 2001;32(8):1832-40.

57. Lahr MMH, Luijckx G-J, Vroomen PCAJ, van der Zee D-J, Buskens E. Proportion of patients treated with thrombolysis in a centralized versus a decentralized acute stroke care setting. Stroke. 2012;43(5):1336-40.

58. Charipar R, Charipar E. Administration of tissue plasminogen activator for acute ischemic stroke in a rural Wisconsin hospital. Wis Med J. 2008;107(4):176-80. 
59. Wang Y, Liao X, Zhao X, Wang DZ, Wang C, Nguyen-Huynh MN, et al. Using recombinant tissue plasminogen activator to treat acute ischemic stroke in China: analysis of the results from the Chinese National Stroke Registry (CNSR). Stroke. 2011:42(6):1658-64.

60. Eriksson M, Jonsson F, Appelros P, Asberg KH, Norrving B, Stegmayr B, et al. Dissemination of thrombolysis for acute ischemic stroke across a nation: experiences from the Swedish stroke register, 2003 to 2008. Stroke. 2010;41(6):1115-22

61. Heuschmann PU, Berger K, Misselwitz B, Hermanek P, Leffmann C, Adelmann $M$, et al. Frequency of thrombolytic therapy in patients with acute ischemic stroke and the risk of in-hospital mortality: the German Stroke Registers Study Group. Stroke. 2003;34(5):1106-13.

62. Jeng J-S, Tang S-C, Deng I-C, Tsai L-K, Yeh S-J, Yip P-K. Stroke center characteristics which influence the administration of thrombolytic therapy for acute ischemic stroke: a national survey of stroke centers in Taiwan. J Neurol Sci. 2009;281:24-7.

63. Kansara A, Chaturvedi S, Bhattacharya P. Thrombolysis and outcome of young stroke patients over the last decade: insights from the Nationwide Inpatient Sample. J Stroke Cerebrovasc Dis. 2013;22(6):799-804.

64. Nilanont Y, Nidhinandana S, Suwanwela NC, Hanchaiphiboolkul S, Pimpak T, Tatsanavivat $P$, et al. Quality of acute ischemic stroke care in Thailand: a prospective multicenter countrywide cohort study. J Stroke Cerebrovasc Dis. 2014;23(2):213-9.

65. Hills NK, Johnston SC. Why are eligible thrombolysis candidates left untreated? Am J Prev Med. 2006;31(6 Suppl 2):S210-6.

66. Bray BD, Campbell J, Cloud GC, Hoffman A, Tyrrell PJ, Wolfe CDA, et al. Bigger, faster?: associations between hospital thrombolysis volume and speed of thrombolysis administration in acute ischemic stroke. Stroke. 2013:44(11):3129-35

67. Engelter ST, Gostynski M, Papa S, Ajdacic-Gross V, Lyrer PA. Barriers to stroke thrombolysis in a geographically defined population. Cerebrovasc Dis. 2007:23(2-3):211-5

68. Moradiya Y, Crystal H, Valsamis H, Levine SR. Thrombolytic utilization for ischemic stroke in US hospitals with neurology residency program. Neurology. 2013;81(23):1986-95.

69. Fang MC, Cutler DM, Rosen AB. Trends in thrombolytic use for ischemic stroke in the United States. J Hosp Med. 2010;5(7):406-9.

70. Madej-Fermo OP, Staff I, Fortunato G, Abbott L, McCullough LD. Impact of emergency department transitions of care on thrombolytic use in acute ischemic stroke. Stroke. 2012;43(4):1067-74.

71. Hoh BL, Chi Y-Y, Waters MF, Mocco J, Barker 2nd FG. Effect of weekend compared with weekday stroke admission on thrombolytic use, in-hospital mortality, discharge disposition, hospital charges, and length of stay in the Nationwide Inpatient Sample Database, 2002 to 2007. Stroke. 2010;41(10):2323-8

72. Keyhani S, Arling G, Williams LS, Ross JS, Ordin DL, Myers J, et al. The use and misuse of thrombolytic therapy within the Veterans Health Administration. Med Care. 2012;50(1):66-73.

73. Iguchi Y, Kimura K, Watanabe M, Shibazaki K, Aoki J. Utility of the Kurashiki prehospital stroke scale for hyperacute stroke. Cerebrovasc Dis. 2011;31 (1):51-6.

74. McKinney JS, Mylavarapu K, Lane J, Roberts V, Ohman-Strickland P, Merlin MA Hospital prenotification of stroke patients by emergency medical services improves stroke time targets. J Stroke Cerebrovasc Dis. 2013:22(2):113-8.

75. Lin CB, Peterson ED, Smith EE, Saver JL, Liang L, Xian Y, et al. Emergency medical service hospital prenotification is associated with improved evaluation and treatment of acute ischemic stroke. Circ Cardiovasc Qual Outcomes. 2012;5(4):514-22.

76. Nagaraja N, Bhattacharya P, Norris G, Coplin W, Narayanan S, Xavier A, et al. Arrival by ambulance is associated with acute stroke intervention in young adults. J Neurol Sci. 2012;316(1-2):168-9.

77. Mullen MT, Kasner SE, Kallan MJ, Kleindorfer DO, Albright KC, Carr BG. Joint commission primary stroke centers utilize more rt-PA in the nationwide inpatient sample. J Am Heart Assoc. 2013;2(2):e000071. doi:10.1161/JAHA.112.000071

78. Joux J, Olindo S, Girard-Claudon A, Chausson N, Saint-Vil M, Signate A, et al. Prehospital transfer medicalization increases thrombolysis rate in acute ischemic stroke. A French stroke unit experience. Clin Neurol Neurosurg. 2013;115(9):1583-5.

79. Ekundayo OJ, Saver JL, Fonarow GC, Schwamm LH, Xian Y, Zhao X, et al, Patterns of emergency medical services use and its association with timely stroke treatment findings from Get With The Guidelines-Stroke. Circ Cardiovasc Qual Outcomes. 2013;6(3):262-9.

\section{Submit your next manuscript to BioMed Central and we will help you at every step:}

- We accept pre-submission inquiries

- Our selector tool helps you to find the most relevant journal

- We provide round the clock customer support

- Convenient online submission

- Thorough peer review

- Inclusion in PubMed and all major indexing services

- Maximum visibility for your research

Submit your manuscript at www.biomedcentral.com/submit
) Biomed Central 